\title{
ON COEFFICIENTS AND ZEROS OF SECTIONS OF POWER SERIES
}

\author{
J. K. SHAW
}

Abstract. For a power series $f(z)=\sum a_{k} z^{k}$ let $s_{n}$ denote the maximum modulus of the zeros of the $n$th partial sum $\sum_{0}^{n} a_{k} z^{k}$. Asymptotic bounds on the sequence $\left|a_{n}\right|^{1 /{ }^{n}} s_{n}$ are obtained for both entire functions and functions with finite radii of convergence. These extend the previous results of J. D. Buckholtz and J. K. Shaw. Finally, conjectures regarding best possible asymptotic bounds are stated.

1. Introduction. For a power series $f(z)=\sum_{k=0}^{\infty} a_{k} z^{k}$, let $s_{n}(f)$ denote the maximum modulus of the zeros of the $n$th section $S_{n}(f ; z)=$ $\sum_{k=0}^{n} a_{k} z^{k}$. If $a_{n}=0$ for some $n$, one conventionally assigns the value $\infty$ to $s_{n}(f)$. This is motivated by the bound

$$
\left|a_{n}\right|^{-1 / n} \leqq s_{n}(f)
$$

which holds whenever $f(0)=1$ and $a_{n} \neq 0$. The quantity on the left is the geometric mean of the moduli of the zeros of $S_{n}(f ; z)$.

Let $c(f)$ denote the radius of convergence of $f$. For $c(f)=1$, define

$$
\rho(f)=\lim \inf s_{n}(f) \text { and } P=\sup _{c(f)=1} \rho(f) ;
$$

$P$, which lies between 1.7818 and 1.82 , is called the power series constant.

It follows from results of a previous paper [2] that the estimate

$$
\lim \inf \left|a_{n}\right|^{1 / n} s_{n}(f) \leqq P
$$

holds for all $f$ with $c(f)<\infty$. The purpose of this note is to obtain, through a highly simplified proof, an improved version of (1.2) and to

Received by the editors October 24, 1972 and, in revised form, December 7, 1972.

AMS (MOS) subject classifications (1970). Primary 30A08, 30A10; Secondary 30A06.

Key words and phrases. Coefficients, zeros of partial sums, the power series constant, entire functions.

(c) American Mathematical Society 1973 
extend this inequality to the case of entire functions. The only result that we require from [2] will be

THEOREM 1. Let $Q(z)=a_{0}+a_{1} z+\cdots+a_{n} z^{n}$ be a polynomial of degree $n$. Then for at least one integer $k, 0 \leqq k \leqq n$, we have

$$
\left|a_{0}+a_{1} z+\cdots+a_{k} z^{k}\right| \geqq\left|a_{n}\right||z|^{k} /(n+1)
$$

for all $|z| \geqq P$.

To simplify the proofs which follow, we will assume throughout that $a_{0}=1$. For functions $f \not \equiv 0$ we have $f(z)=a_{m} z^{m} f_{1}(z), f_{1}(0)=1$, and $s_{n}(f)=$ $s_{n-m}\left(f_{1}\right)$. In applications, the loss of generality thus amounts to a uniform shift of indices.

2. The maximum term in a power series. Let $\sum_{k=0}^{\infty} a_{k} z^{k}$ be a power series and define

$$
d_{n}=\left.\max _{0 \leqq j<n}\left|a_{j}\right| a_{n}\right|^{1 /(n-j)} \quad\left(a_{n} \neq 0\right) .
$$

Thus $\left|a_{n}\right| d_{n}^{n} \geqq\left|a_{k}\right| d_{n}^{k}, 0 \leqq k \leqq n$, and $d_{n}$ is the smallest number with this property. For notational purposes, we find it convenient to assign the value $\infty$ to each of the expressions $\left|a_{n}\right|^{1 / n} d_{n}$ and $\left|a_{n}\right|^{1 / n} s_{n}(f)$ whenever $a_{n}=0$.

THEOREM 2. Suppose $f(z)=\sum_{k=0}^{\infty} a_{k} z^{k}$ is not a polynomial and let $\alpha=\lim \inf \left|a_{n}\right|^{1 / n} d_{n}$. Then

$$
1 \leqq \lim \inf \left|a_{n}\right|^{1 / n} s_{n}(f) \leqq P \alpha .
$$

Proof. Let $n$ be a nonnegative integer such that $a_{n} \neq 0$, let $\varepsilon>0$ and let $\lambda=d_{n}(1+\varepsilon)$. If, in (1.3), we replace $a_{j}$ by $a_{j} \lambda^{j}$ and then $z$ by $z / \lambda$, we obtain

$$
\left|a_{0}+a_{1} z+\cdots+a_{k} z^{k}\right| \geqq \lambda^{n-k}\left|a_{n}\right||z|^{k} /(n+1),
$$

for some $k, 0 \leqq k \leqq n$, and all $|z| \geqq P \lambda$. Let $k(n)$ denote the least $k$ for which (2.2) holds. Noting that (2.2) holds for arbitrarily large $|z|$, one sees that $\left|a_{k(n)}\right| \geqq\left|a_{n}\right|\left(d_{n}(1+\varepsilon)\right)^{n-k(n)} /(n+1)$. Therefore

$$
d_{n}^{n-k(n)} \geqq d_{n}^{n-k(n)}(1+\varepsilon)^{n-k(n)} /(n+1),
$$

and it follows that $k(n) / n \rightarrow 1$.

Since the left member of (2.2) does not vanish for $|z| \geqq P \lambda$, then $s_{k(n)}(f) \leqq P d_{n}(1+\varepsilon)$. In view of $\left|a_{k(n)}\right| d_{n}^{k(n)} \leqq\left|a_{n}\right| d_{n}^{n}$, it follows that

$$
\left|a_{k(n)}\right|^{1 / k(n)} s_{k(n)}(f) \leqq\left[\left|a_{n}\right|^{1 / n} d_{n}\right]^{n / k(n)} P(1+\varepsilon) .
$$


There exists, then, an infinite set $J$ of integers such that $j \in J$ implies

$$
\left|a_{k(j)}\right|^{1 / k(j)} s_{k(j)}(f) \leqq(\alpha+\varepsilon)^{(1+\varepsilon)} P(1+\varepsilon),
$$

and the right-hand side of (2.1) now follows easily.

The left-hand side of (2.1) follows from (1.1), and this completes the proof.

LeMma 1. Suppose that $f(z)=\sum_{k=0}^{\infty} a_{k} z^{k}$ has finite radius of convergence. Then

$$
\lim \inf \left|a_{n}\right|^{1 / n} d_{n}=1 .
$$

PRoOF. Suppose first that $c(f)>0$. If $r>c(f)$, then the sequence $\left\{a_{n} r^{n}\right\}_{0}^{\infty}$ is unbounded. Thus $\left|a_{k}\right| r^{k} \geqq\left|a_{j}\right| r^{j}, 0 \leqq j \leqq k$, for infinitely many $k$. For such $k$ we have $r \geqq\left.\left|a_{j}\right| a_{k}\right|^{1 /(k-j)}, 0 \leqq j \leqq k$, and so $r \geqq d_{k}$. Therefore

$$
\lim \inf \left|a_{n}\right|^{1 / n} d_{n} \leqq\left(\lim \sup \left|a_{n}\right|^{1 / n}\right)\left(\lim \inf d_{n}\right) \leqq r / c(f) .
$$

Letting $r \rightarrow c(f)$, we get lim inf $\left|a_{n}\right|^{1 / n} d_{n} \leqq 1$.

If $c(f)=0$, then $\left|a_{n}\right|^{1 / n} \geqq\left|a_{j}\right|^{1 / j}, 0 \leqq j \leqq n$, for infinitely many $n$. This implies

$$
\left|a_{n}\right|^{1 / n}\left|a_{j} / a_{n}\right|^{1 /(n-j)}=\left[\left|a_{j}\right| /\left|a_{n}\right|^{j / n}\right]^{1 /(n-j)} \leqq 1,
$$

and therefore $\left|a_{n}\right|^{1 / n} d_{n} \leqq 1$. Hence $\lim \inf \left|a_{n}\right|^{1 / n} d_{n} \leqq 1$.

For the opposite inequality, note that $\left|a_{n}\right| d_{n}^{n} \geqq\left|a_{0}\right| d_{n}^{0}=1$, for all $n$, and so $\lim \inf \left|a_{n}\right|^{1 / n} d_{n} \geqq 1$.

COROLlaRY 1. If $f(z)=\sum_{k=0}^{\infty} a_{k} z^{k}$ has finite radius of convergence, then

$$
1 \leqq \lim \inf \left|a_{n}\right|^{1 / n} s_{n}(f) \leqq P .
$$

REMARK. Equality occurs on the left-hand side of (2.3) for the function $(1-z)^{-1}$. Buckholtz [1] has constructed an example which shows that $P$ cannot be replaced in (2.3) by a number less than 1.7818. The conjecture that $P$ is the sharp constant remains unsettled.

If $f(z)=\sum_{k=0}^{\infty} a_{k} z^{k}$ is a transcendental entire function, let $\mu(r)$ and $v(r)$ denote the maximum term and central index, respectively, of the series for $f$. Let $N=\left\{n: a_{n} \neq 0\right\}$ and let $R$ denote the range of $v$. Then $R \subset N$. For each $n \in R$, let $r_{n}$ be the least number $r$ for which $v(r)=n$. The estimate $\left|a_{n}\right| r_{n}^{n} \geqq\left|a_{j}\right| r_{n}^{j}$ holds for all $j$, and strict inequality holds for $j>n$. In particular $r_{n} \geqq d_{n}$, for $n \in R$.

If $n \in R$ then $v\left(r_{n}\right)=n$ and $\mu\left(r_{n}\right)=\left|a_{n}\right| r_{n}^{n}$. Therefore

$$
\begin{aligned}
\liminf _{r \rightarrow \infty}[\mu(r)]^{1 / v(r)} & =\liminf _{n \in R}\left[\mu\left(r_{n}\right)\right]^{1 / v\left(r_{n}\right)}=\liminf _{n \in R}\left|a_{n}\right|^{1 / n} r_{n} \\
& \geqq \liminf _{n \in R}\left|a_{n}\right|^{1 / n} d_{n} \geqq \liminf _{n \in N}\left|a_{n}\right|^{1 / n} d_{n} \\
& =\liminf \left|a_{n}\right|^{1 / n} d_{n} .
\end{aligned}
$$


S. M. Shah [4] has shown that if $f$ has order $\rho>0$, then

$$
\liminf _{r \rightarrow \infty}[\mu(r)]^{1 / v(r)} \leqq e^{1 / \rho} .
$$

From Theorem 2 we thus obtain

COROLlaRY 2. If $f(z)=\sum_{k=0}^{\infty} a_{k} z^{k}$ is a transcendental entire function of order $\rho>0$, then

$$
1 \leqq \lim \inf \left|a_{n}\right|^{1 / n} s_{n}(f) \leqq P e^{1 / \rho} .
$$

Remarks. (i) There exist [2] gap series $\sum_{j=0}^{\infty} z^{n_{3}} /\left(n_{j}\right)$ ! such that equality holds on the left side of (2.4). As in Corollary 1 , the conjecture that $P$ is the sharp constant in (2.4) seems to be true.

(ii) From Corollaries 1 and 2 we obtain the following result: if the formal power series $\sum_{0}^{\infty} a_{k} z^{k}$ satisfies $\lim \inf \left|a_{n}\right|^{1 / n} s_{n}(f)=\infty$, then $\sum_{0}^{\infty} a_{k} z^{k}$ is the power series for an entire function of order zero. The reader may wish to compare this with J. Korevaar's similar result [3] on angular distribution of zeros: let $S$ be a fixed sector with vertex 0 , and let $N(S, n)$ denote the number of zeros of $S_{n}(f ; z)$ in $S$; if $N(S, n)=o(n)$, then $f$ is an entire function of order zero.

(iii) Throughout this paper, the condition $a_{0}=1$ can be replaced by $c(f)>0$, with the same results holding.

\section{REFERENCES}

1. J. D. Buckholtz, Zeros of partial sums of power series, Michigan Math. J. 15 (1968), 481-484. MR 38 \#3409.

2. J. D. Buckholtz and J. K. Shaw, Zeros of partial sums and remainders of power series, Trans. Amer. Math. Soc. 166 (1972), 269-284.

3. J. Korevaar, The zeros of approximating polynomials and the canonical representation of an entire function, Duke Math. J. 18 (1951), 573-592. MR 13, 222.

4. S. M. Shah, The maximum term of an entire series, Math. Student 10 (1942), 80-82. MR 4, 137.

Department of Mathematics, Virginia Polytechnic Institute and State UNIVERSITY, BLACKSBURG, VIRGINIA 24061 